\title{
Nanoridges for single-photon emission
}

Quantum technologies require the precise control of individual quantum systems, which can be achieved using trains of identical, coherent photons. Further control can be achieved if these single photons also have well-defined polarization properties. To date, polarized single-photon sources have used a quantum emitter exposed to very high magnetic fields at cryogenic temperatures, which are challenging to scale out of the lab and

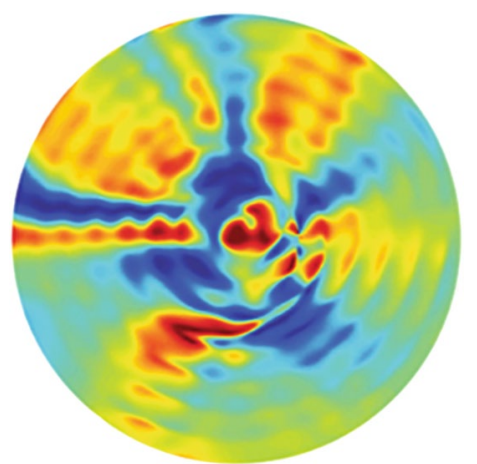

Credit: Adapted with permission from Kan et al., Adv. Mater. https://doi.org/10.1002/adma. 201907832 (2020), Wiley. into real-world applications. Writing in Advanced Materials, Yinhui Kan and colleagues present a new set-up that works without an external magnet and at room temperature.

Single photons are generated by shining a laser on to a quantum emitter, such as a nanodiamond with a nitrogen-vacancy centre. In the device developed by Kan et al., the nitrogen-vacancy centre is placed in the middle of an optical metasurface on $\mathrm{SiO}_{2}-\mathrm{Ag}$ substrate. When the nitrogen-vacancy centre is excited by a radially polarized laser, the emitted photons interact with the substrate, forming surface plasmon-polariton modes. These modes then in turn interact with the optical metasurface composed of a series of concentric circular nanoridges. The key to achieving circularly polarized emitted photons is in the varying width of the ridges. Changing the width of the ridge along the circumference of the circle induces a change in the relative phase shift of the electric field along the circle. This varying phase shift makes the electric field scatter in such a way that circularly polarized single photons are emitted.

The device architecture was first developed using simulations (pictured) to find the optimal parameters, which were then implemented experimentally. In devices without nanoridges, the emitted light had no circular polarization. With the introduction of the nanoridges, the emission both increased in intensity (owing to more light being scattered from the ridges to the detectors) and also became circularly polarized. The devices must be carefully fabricated, but by removing the need for high magnetic fields and very low temperatures, they may lead to practical applications in quantum communication.

Ankita Anirban

ORIGINAL ARTICLE Kan, Y. et al. Metasurfaceenabled generation of circularly polarized single photons. Adv. Mater. https://doi.org/10.1002/ adma.201907832 (2020) 